\title{
Continuous growth of vimentin filaments in mouse fibroblasts
}

\author{
THOMAS R. COLEMAN* and ELIAS LAZARIDES $\dagger$ \\ Division of Biology, California Institute of Technology, Pasadena, CA 91125, USA \\ *Author for correspondence \\ $\dagger$ Present address: Building 45-333, Merck Sharp and Dohme Research Laboratories, West Point, PA 19486, USA
}

\section{Summary}

We have investigated the dynamics of intermediate filament assembly in vivo by following the fate of heterologous chicken vimentin subunits expressed under the control of an inducible promoter in transfected mouse fibroblasts. Using RNase protection, metabolic protein pulse-chase and immunofluorescence microscopy, we have examined the fate of newly assembled subunits under physiological conditions in situ. Following induction and subsequent removal of inducer, chicken vimentin mRNA had a half-life of approximately $6 \mathrm{~h}$ while both chicken and mouse vimentin protein polymer had long half-lives - roughly equivalent to the cell generation time. Moreover, following deinduction, chicken vimentin immunolocalization progressed from a continuous (8-10 $\mathrm{h}$ chase) to a discontinuous $(\geq 20$ h chase) pattern. The continuous chicken vimentin staining reflects the uniform incorporation of chicken vimentin throughout the endogenous mouse vimentin network while the discontinuous or punctate chicken vimentin staining represents short interspersed segments of assembled chicken vimentin superimposed on the endogenous polymer. This punctate staining pattern of chicken vimentin was present throughout the entire array of intermediate filaments, with no bias toward the perinuclear region. These results are consistent with a continuous growth model of intermediate filament assembly, wherein subunit addition occurs at discrete sites located throughout the cytoskeleton.

Key words: vimentin, intermediate filaments, assembly dynamics.

\section{Introduction}

The cytoskeleton in higher eukaryotes is composed of three major filament classes: actin, microtubules and intermediate filaments (IFs). Together with associated proteins, these filaments establish the internal architecture of cells. Of these three filament systems, the least is known about IF assembly characteristics and functional roles in vivo (for reviews see Steinert and Roop, 1988; Klymkowsky et al., 1989; Stewart, 1990). Owing to their relative insolubility in physiological conditions and small soluble subunit pool in vivo, IFs are often depicted as inert stable cytoskeletal components.

Recent work, however, has clearly demonstrated that IFs are, in fact, highly dynamic structures (reviewed by Steinert and Liem, 1990; Skalli and Goldman, 1991). In vitro fluorescence resonance energy-transfer studies have demonstrated that the neurofilament polymer is in dynamic equilibrium with a small but kinetically active unassembled subunit pool (Angelides et al., 1989). Other studies have suggested a dynamic phosphorylation-mediated reversible disassembly of a wide variety of IFs including: nuclear lamins (Gerace and Blobel, 1980), neurofilaments (Hisanaga et al., 1990), desmin (Evans, 1988a; Geisler and Weber, 1988; Inagaki et al., 1988) and vimentin (Inagaki et al., 1987; Evans, 1988a,b). Even though most of these aforementioned studies have been carried out in vitro, such a phosphorylation-mediated IF assembly/disassembly mechanism likely plays an important role in vivo as well. Relatedly, microinjection of A-kinase into fibroblasts causes phosphorylation of specific vimentin moieties coincident with vimentin network collapse (Lamb et al., 1989). Furthermore, several reports have implicated cdc2 (an enzyme playing a key role in the regulation of mitosis) as the kinase responsible for site-specific lamin phosphorylation (Heald and McKeon, 1990; Peter et al., 1990; Ward and Kirschner, 1990) and vimentin phosphorylation (Chou et al., 1990). Thus, both A-kinase and cdc2 kinase may regulate IF assembly dynamics in vivo through phosphorylation of specific residues.

Further evidence demonstrating IF assembly dynamics comes from transfection studies in which genes encoding tagged IF subunits were mutated, transfected and the resultant mutant proteins were assayed for assembly competence by immunofluorescence. This basic approach has been used by several groups to delineate structural and sequence requirements for IF assembly. Assembly-incompetent mutant neurofilament (Monteiro and Cleveland, 1989; Gill et al., 1990; Wong and Cleveland, 1990), vimentin (Christian et al., 1990), desmin (Raats et al., 1990) and keratin (Albers and Fuchs, 1987, 1989; Lu and Lane, 1990) subunits caused the breakdown of the entire filament network, suggesting that newly synthesized mutant subunits enter the pre-existing endogenous polymer, thereby facilitating its 
subsequent disintegration. Moreover, rapid assembly of vimentin into the pre-existing network has also been demonstrated by following the induced expression of exogenous subunits in mouse fibroblasts (Ngai et al., 1990) and HeLa cells (Sarria et al., 1990).

While it is clear that IF assembly is indeed a dynamic process in vivo, several important questions remain regarding the fate of the newly assembled subunits. Do regions of the IF polymer have differential stabilities or is subunit loss from IFs minimal? Resolution of these issues requires an experimental system in which the fate of newly assembled vimentin protein can be examined. To this end, mouse Balb/c 3T3 fibroblasts were stably transfected with a chimeric plasmid containing the chicken vimentin gene under the control of the glucocorticoid-inducible mouse mammary tumor virus long terminal repeat (Ngai et al., 1990). Using this system, we have analyzed the temporal and spatial dynamics of inducible IF mRNA and protein turnover following deinduction of chicken vimentin by RNase protection, metabolic labeling and immunofluorescence microscopy. Chicken vimentin mRNA has a half-life of $6 \mathrm{~h}$ and vimentin polymer has a half-life approximating that of cell generation time. Furthermore, during the first $28 \mathrm{~h}$ after deinduction, chicken vimentin protein dramatically reverses its immunolocalization progression. Taken together, these findings are consistent with a continuous growth model of vimentin assembly, wherein subunits are added at discrete sites throughout the cytoplasm.

\section{Materials and methods}

\section{Cell lines, cell culture and dexamethasone induction}

The construction, transfection and initial characterization of both the inducible chicken vimentin (3T3MTV2-108) and the constitutively expressing chicken vimentin (3T3XBB-5) cell lines have been described previously (Ngai et al., 1990). These stably transfected chicken vimentin-expressing mouse fibroblasts were maintained under constant G418 selection $(400 \mu \mathrm{g} / \mathrm{ml})$. Balb/c 3T3 cells and their derivatives were maintained in Dulbecco's modified Eagle medium (DMEM) supplemented with 10\% heat-inactivated fetal bovine serum and penicillin/streptomycin. For induction/deinduction experiments, cells were exposed to supplemented medium containing $5 \times 10^{-7} \mathrm{M}$ dexamethasone for $4 \mathrm{~h}$, washed with $\mathrm{Ca}^{2+}$ - and $\mathrm{Mg}^{2+}$-free Earle's buffered salt solution (EBSS) and returned to supplemented medium (without dexamethasone) for the indicated chase times.

\section{Immunofluorescence microscopy and statistical analysis}

$3 \mathrm{~T} 3 \mathrm{MTV}^{2}-108$ cells grown on acid-treated glass coverslips were fixed, permeabilized and processed for immunofluorescence microscopy as described previously (Granger et al., 1982). Fixed cells were then incubated sequentially with chicken vimentinspecific monoclonal antibody 10D1/D11 (1:9 dilution of culture supernatant), RITC-conjugated rabbit anti-mouse $\mathrm{IgG}$, normal mouse serum, goat anti-vimentin antibody (1:50; Polysciences, Inc., Warrington, PA) and normal mouse serum-absorbed FITCconjugated rabbit anti-goat IgG. Cells were viewed with Leitz Orthoplan epifluorescence ( $63 \times$ objective) and photographed on Kodak Tri-X 400 film using an automatic exposure meter.

The effect of induction/deinduction on populations of $3 \mathrm{~T} \mathrm{MTV}^{2}-108$ cells was determined using immunofluorescence by scoring each chicken vimentin-positive cell as either discon- tinuous (Fig. 3C) or continuous (Fig. 3E) staining. To avoid experimental bias, immunofluorescence studies were coded, shuffled, scored "blind," and then decoded. The percentage of discontinuous vimentin staining cells were pooled from the following chase times: group 1, 0-6 h; group 2, 8-10 h; group 3, $\geq 20 \mathrm{~h}$. The Bonferroni procedure for multiple comparisons (McClave and Dietrich, 1988) was used to test which groups differed from each other. Data from 8 separate induction/deinduction experiments, representing over 3000 cells, were pooled and compared.

\section{RNA isolation and quantitative RNase protection}

Total cellular RNA was isolated by the guanidinium thiocyanate lysis method (Chirgwin et al., 1979). Homogenates were layered over cushions of $5.7 \mathrm{M} \mathrm{CsCl}, 1 \mathrm{mM}$ EDTA, pH 8.0, and RNA was pelleted by centrifugation (Sambrook et al., 1989). Steadystate chicken and murine vimentin mRNA levels were assayed by protection of a $1.9 \mathrm{~kb}{ }^{32} \mathrm{P}$-labeled complementary RNA generated with bacteriophage T7 polymerase (Zinn et al., 1983). Correctly initiated chicken vimentin mRNA was expected to protect 632 nucleotides (nt) (corresponding to exon 1) of the labeled probe. Typically, $1-2$ ng [32P]RNA probe $\left(3 \times 10^{5} \mathrm{cts} / \mathrm{min}\right)$ was hybridized to $10 \mu \mathrm{g}$ total cellular RNA. Hybridization, digestion conditions and quantitation of protected fragments were performed exactly as described previously (Ngai et al., 1987).

\section{$\left[{ }^{35}\right.$ S $]$ methionine labeling and immunoblotting}

Plated, adherent cells were washed twice with EBSS and incubated $40 \mathrm{~min}$ at $37^{\circ} \mathrm{C}$ with or without $5 \times 10^{-7} \mathrm{M}$ dexamethasone in methionine-depleted MEM (Flow Laboratories, McLean, VA) supplemented with 5\% dialyzed fetal bovine serum. For each condition, $35 \mu \mathrm{Ci} / \mathrm{ml}\left[{ }^{35} \mathrm{~S}\right]$ methionine was added and cells were pulselabeled for $20 \mathrm{~min}$ at $37^{\circ} \mathrm{C}$. For the induction/deinduction experiments, cells were pulse-labeled with $\left[{ }^{35} \mathrm{~S}\right]$ methionine for the last $20 \mathrm{~min}$ of the $4 \mathrm{~h}$ induction period, washed to remove unincorporated $\left[{ }^{35} \mathrm{~S}\right]$ methionine and harvested after incubation for the indicated chase times $(0-28 \mathrm{~h})$. Cells were washed and removed from their plates by trypsin treatment and fractionated into soluble and cytoskeletal pools using the Triton X-100/urea buffers of Moon and Lazarides (1983). A sample of the trypsin-treated cells was used to determine cell doubling time. Equivalent amounts of ${ }^{35} \mathrm{~S}$-labeled cytoskeletal protein (as determined by TCA precipitation; Ngai et al., 1984) were resolved on $12.5 \%$ SDS-polyacrylamide gels. Gels were either stained with Coomassie blue to visualize total protein and then impregnated with 2,5-diphenyloxazole for fluorography (Bonner and Laskey, 1974) or transferred to nitrocellulose (Coleman et al., 1987) and incubated with chicken vimentin-specific monoclonal 10D1/D11 antibody. Immunoreactive bands were visualized by secondary staining with alkaline phosphatase-conjugated anti-mouse IgG according to the manufacturer's recommendations (BioRad Laboratories, Richmond, CA).

\section{Protein quantitation and radioactivity determination}

Quantitation of both vimentin and actin in cytoskeletal extracts was determined by scanning the gels using a densitometer (E-C Apparatus Corporation, St. Petersburg, FL) linked to an integrator (Hewlett Packard model 3390A, Fullerton, CA). Vimentin and actin gel bands routinely contained $0.5-1.0 \mu \mathrm{g}$, which was well within the linear range $(0.2-2.0 \mu \mathrm{g})$ of Coomassie blue binding for identically treated purified vimentin and actin standards. For every experiment requiring quantitation, the sample containing the highest protein concentration was run in parallel at multiple loadings (1.4 to 0.2 equivalents) to determine whether all experimental loads were in the linear range of Coomassie blue binding and scintillation emission (see below).

Total cytoskeletal protein radioactivity was determined by 
trichloroacetic acid (TCA) precipitation and scintillation counting (Ngai et al., 1987). Vimentin and actin radioactivity levels were determined by excising, hydrating and counting the relevant gel bands (Blikstad and Lazarides, 1983). For the protein standard curves, chicken breast muscle actin was prepared from acetone powder according to the methods of Spudich and Watt (1971). Bovine or porcine lens vimentin was purified from urea extracts (Nelson and Traub, 1982) by gel filtration (Aca 24 column; Pharmacia LKB Biotechnology, Piscataway, $\mathrm{NJ}$ ) in $10 \mathrm{mM}$ Tris- $\mathrm{HCl}$, $\mathrm{pH}$ 7.5, $6 \mathrm{M}$ urea, $1 \mathrm{mM}$ EGTA, $1 \mathrm{mM}$ dithiothreitol, 40 t.i.u. aprotinin, $0.1 \mathrm{mM}$ PMSF, $0.02 \% \mathrm{NaN}_{3}$. Vimentin-containing fractions were pooled, ammonium sulfate precipitated and purified to homogeneity on an hydroxyapatite column (Vikstrom et al., 1989). Protein concentrations were determined by the method of Bradford (1976) or using the G-actin $A_{280}^{1 \%}$ of $10.9 / \mathrm{cm}$.

\section{Results}

Steady-state levels of induced chicken vimentin mRNA decline slowly after dexamethasone removal

Since the level of vimentin filament expression is regulated primarily by mRNA abundance (Ngai et al., 1984), in order to understand the exit kinetics of induced vimentin protein we first examined the deinduction kinetics of vimentin mRNA. We performed these experiments using the 3T3MTV ${ }^{2}-108$ mouse fibroblast cell line, which contains an inducible chicken vimentin construct. We induced 3T3MTV ${ }^{2}-108$ cells for $4 \mathrm{~h}$ with dexamethasone, then chased in the absence of dexamethasone for varying times and isolated total cellular RNA. As expected, RNase protection of control (nontransfected parental) Balb/c 3T3 RNA demonstrated no chicken vimentin-protected band (Fig. 1A, lane 2). This probe did hybridize to mouse vimentin RNA, generating the 70 and 80 nt protected fragments present in all murine fibroblast derivatives (Fig. 1A, lanes 2-11) but not in tRNA control (Fig. 1A, lane 1). Positive control chicken vimentin RNase protections of 3T3XBB-5 RNA (these cells constitutively express chicken and mouse vimentin) yielded the expected $632 \mathrm{nt}$ chicken exon 1 fragment (Fig. 1A, lanes 10 and 11). RNase protections of two concentrations of 3T3XBB-5 RNA demonstrated the quantitative nature of these protection reactions in terms of both chicken and mouse vimentin-protected fragments (compare chicken vimentin and mouse vimentin band intensities; Fig. 1A, lanes 10 and 11). Uninduced
3T3MTV $2-108$ cells expressed a low but detectable level of correctly initiated chicken vimentin mRNA (Fig. 1A, lane 3). After a $4 \mathrm{~h}$ dexamethasone induction, 3T3MTV2. 108 cells displayed a 4.3-fold increase in chicken vimentin RNA, which declined back to uninduced levels by $\sim 12-16$ $\mathrm{h}$ when chased without dexamethasone (Fig. 1B); quantitation of gel bands revealed a chicken vimentin mRNA halflife of $\sim 6 \mathrm{~h}$ (Fig. 1B). By comparison, the mouse vimentinprotected fragment remained roughly constant throughout the deinduction chase period (Fig. 1A, lanes 4-9). Consistent with a chicken vimentin mRNA half-life of $\sim 6 \mathrm{~h}$ is the fact that no newly translated chicken vimentin protein was detectable by $\left.{ }^{35} \mathrm{~S}\right]$ methionine labeling and fluorography 12-16 $\mathrm{h}$ after the termination of a $4 \mathrm{~h}$ induction (data not shown).

Chicken and mouse vimentin proteins are stable cytoskeletal components with half-lives approximating that of cell division

To gain a more complete understanding of the fate of newly assembled vimentin and to assess the relative amounts of chicken and mouse vimentin produced in these cells, additional induction/deinduction experiments were performed using $\left[{ }^{35} \mathrm{~S}\right]$ methionine to monitor protein levels. 3T3MTV ${ }^{2}$ 108 cells were pulsed with $\left[{ }^{35}\right.$ S $]$ methionine during the last $20 \mathrm{~min}$ of the $4 \mathrm{~h}$ induction and equivalent amounts of radioactivity from cytoskeletal samples were loaded onto SDS-polyacrylamide gels. With the gel system employed here, chicken vimentin migrates with a slightly higher relative mobility compared to mouse vimentin, as determined previously by two-dimensional gel analysis (Ngai et al., 1987). By loading equivalent counts, we expect the later chase times to have more total protein per cts/min as a result of cell division in nonradioactive medium. When visualized with Coomassie blue, the amount of mouse vimentin and actin did increase with chase time (Fig. 2A, Coomassie; compare mouse vimentin and actin band intensities of lanes 1-4 to lanes 7 and 8). Quantitation of protein and radioactivity levels revealed that the specific activities of both actin and mouse vimentin declined with half-lives of 26 and 32 $\mathrm{h}$, respectively (Table 1). For comparison, the specific activity of the total cytoskeletal protein declined with a halflife of $29 \mathrm{~h}$, while the cell doubling time was $28 \mathrm{~h}$ (Table 1). Chicken vimentin was not visualized by Coomassie stain but ${ }^{35} \mathrm{~S}$-incorporated chicken vimentin bands were readily observed upon induction (Fig. 2A, Autorad., compare lanes

Table 1. Mouse and chicken vimentin are stable cytoskeletal components which have half-lives approximating that of the cell doubling time

\begin{tabular}{|c|c|c|c|c|c|}
\hline \multirow[b]{2}{*}{ Chase period $(\mathrm{h}) \dagger$} & \multirow[b]{2}{*}{ Cell number $\ddagger$} & \multicolumn{4}{|c|}{ Specific activity (cts/min per $\mu \mathrm{g})^{*}$} \\
\hline & & Total cytoskeletal protein & Actin & Mouse vimentin & Chicken vimentin \\
\hline 0 & 290,000 & $2.9 \times 10^{6}\left(2.2 \times 10^{4}\right)$ & $18800(1900)$ & $2070(380)$ & $3350(140)$ \\
\hline 9 & 410,000 & $2.4 \times 10^{6}\left(1.9 \times 10^{4}\right)$ & $14500(4800)$ & $1660(46)$ & $2150(230)$ \\
\hline 28 & 570,000 & $1.5 \times 10^{6}\left(5.1 \times 10^{3}\right)$ & $8600(940)$ & $1160(160)$ & $1790(180)$ \\
\hline Doubling time $(\mathrm{h}) \S$ & 28 & - & - & - & - \\
\hline Half-life $(\mathrm{h}) \S$ & - & 29 & 26 & 32 & 31 \\
\hline \multicolumn{6}{|c|}{$\begin{array}{l}\text { *Each value represents an average of } 4 \text { determinations and is expressed as cts/min per } \mu \mathrm{g} \text {; one standard deviation is indicated in parentheses. } \\
\dagger \text { In the absence of }\left[{ }^{35} \mathrm{~S}\right] \text { methionine. } \\
\ddagger \text { Per } 100 \mathrm{~mm} \text { plate. } \\
\text { §As determined from linear regression. }\end{array}$} \\
\hline
\end{tabular}



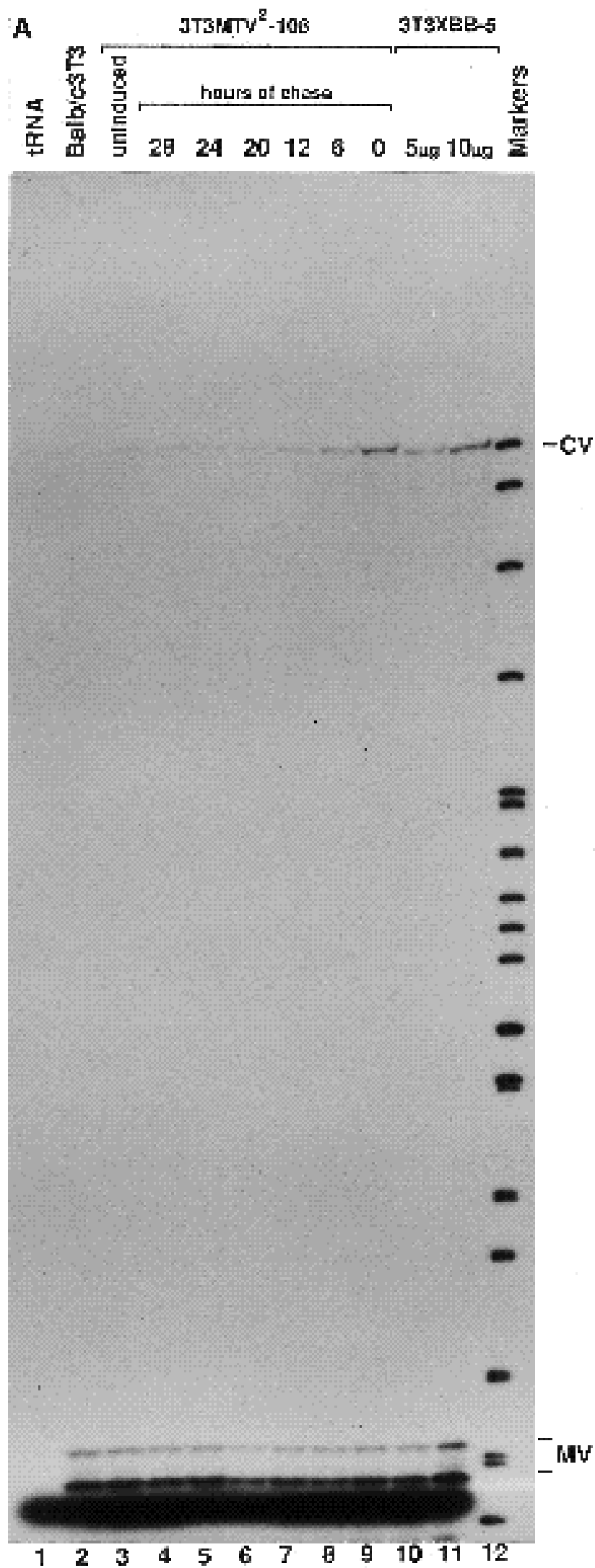

1 and 2 with lanes 3 and 4). Like the relatively stable mouse vimentin, chicken vimentin-specific activity declined with a half-life of $31 \mathrm{~h}$ (Table 1). Moreover, total chicken

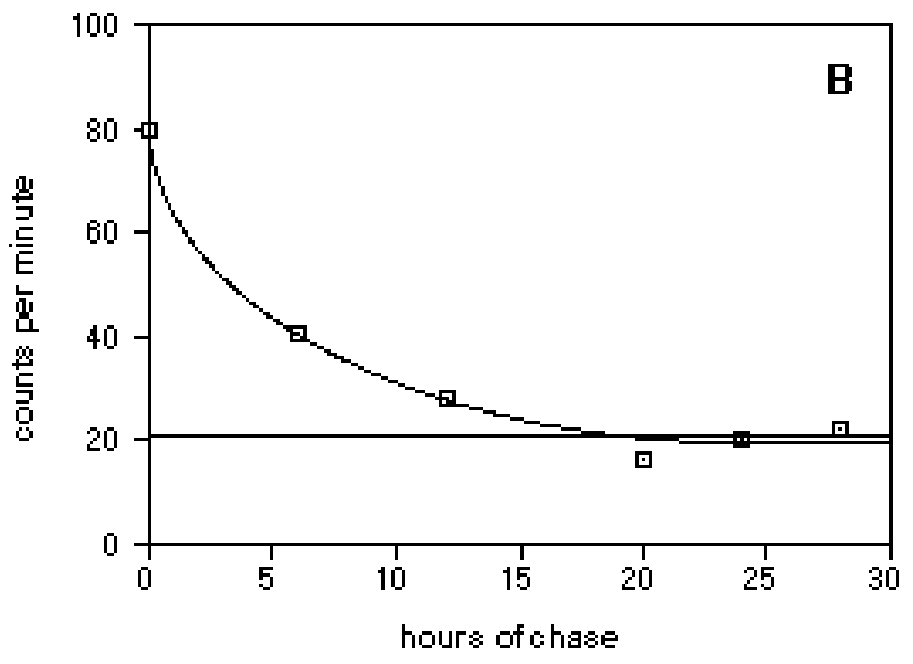

Fig. 1. Analysis of chicken vimentin mRNA levels following induction and deinduction. (A) A ${ }^{32}$ P-labeled RNA probe complementary to chicken vimentin message was hybridized to total cellular RNA and subjected to RNase digestion. Control lanes 1 and 2 show protection of probe from RNase digestion after hybridization with $10 \mu \mathrm{g}$ yeast tRNA and $10 \mu \mathrm{g}$ RNA isolated from untransfected Balb/c 3T3 cells, respectively. Lanes 3-9 show protection of probe after hybridization with $10 \mu \mathrm{g}$ RNA isolated from 3T3MTV $2-108$ cells which were uninduced (lane 3 ) or induced for $4 \mathrm{~h}$ and chased in the absence of dexamethasone for $28,24,20,12,6,0 \mathrm{~h}$ (lanes 4-9), respectively. Lanes 10 and 11 show protection of probe after hybridization with 5 and $10 \mu \mathrm{g}$ RNA isolated from Balb/c 3T3 cells stably transfected with the entire chicken vimentin gene (3T3XBB-5). Lane 12 shows the migration pattern of ${ }^{32} \mathrm{P}$-labeled pBR322-HpaII DNA markers (Markers). Fragments corresponding to the 70 and $80 \mathrm{nt}$ mouse vimentin (MV) and the $632 \mathrm{nt}$ chicken vimentin exon 1 (CV) are indicated. Note that the mouse vimentin protected fragments serve as an internal standard of RNA load, remaining relatively constant in lanes 1-9 and reflecting the doubling of load between lanes 10 and 11. (B) Quantitation of chicken vimentin specific bands shown in Fig. 1A reveals a 4.3-fold induction of chicken vimentin mRNA immediately following induction and a chicken vimentin mRNA half-life of $\sim 6 \mathrm{~h}$. Horizontal line indicates the background level of uninduced 3T3MTV 2 -108 chicken vimentin-specific radioactivity. Both plots have been corrected for background radioactivity.

vimentin protein increased slightly from $0-9 \mathrm{~h}$ chase (before mRNA degradation) but then remained relatively constant (Fig. 2A, Immunoblot; compare lanes 3 and 4 with 5 and 


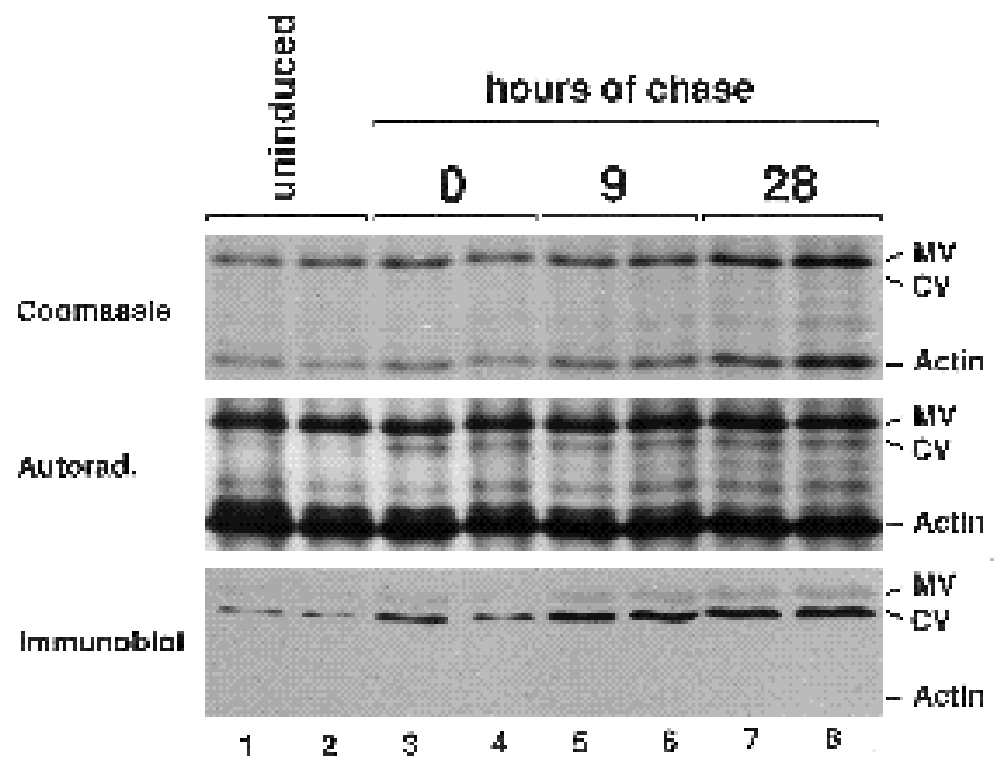

Fig. 2. Mouse and chicken vimentin proteins are stable cytoskeletal components which display half-lives approximating that of cell division. 3T3MTV $2-108$ cells were labeled for $20 \mathrm{~min}$ with $\left[{ }^{35} \mathrm{~S}\right]$ methionine in the absence of dexamethasone (uninduced lanes 1 and 2 ) or for the last $20 \mathrm{~min}$ of a $4 \mathrm{~h}$ induction period (lanes 3-8). At each time point, duplicate samples were prepared from identically treated cellular populations. Cells were harvested immediately following the $\left[{ }^{35} \mathrm{~S}\right]$ methionine pulse (lanes 1 and 2 ) or after a chase period in the absence of radioactive methionine and dexamethasone; chase times are indicated above the lanes $(0 \mathrm{~h}$, lanes 3 and 4; $9 \mathrm{~h}$, lanes 5 and 6 ; and $28 \mathrm{~h}$, lanes 7 and 8). Equivalent total counts of cytoskeletal samples were loaded onto SDS-polyacrylamide gels and visualized according to total protein (Coomassie blue stained: Coomassie, upper panel), radioactive protein (Autoradiography: Autorad., middle panel) or total chicken vimentin protein (10D1/D11 immunoblot: Immunoblot, lower panel). Each panel shows only the middle portion of the gel; the relative mobilities of mouse vimentin (MV), chicken vimentin (CV) and actin (Actin) are indicated. Top and middle panels show regions of the same gel, stained first with Coomassie blue, then processed for fluorography. When loaded according to equivalent ${ }^{35} \mathrm{~S}$-incorporated counts, note how total protein increases with chase time (as indicated by increase in intensities of both mouse vimentin and actin bands), consistent with decreasing specific activity due to cell growth (Coomassie, upper panel). Chicken vimentin cannot be visualized by Coomassie blue stain, but radioactive chicken vimentin is clearly visible following dexamethasone induction (Autorad., middle panel). Similarly, total chicken vimentin increases dramatically upon induction and slightly from $0-9 \mathrm{~h}$ chase, then changes little with subsequent chase (Immunoblot, lower panel).

6, and 7 and 8). Taken together, the data presented in Fig. 2 and Table 1 suggest that both mouse and chicken vimentin are stable cytoskeletal components with half-lives approximating that of the cell generation time.

\section{Vimentin immunolocalization progresses from a continuous to a discontinuous staining pattern following deinduction}

To determine the intracellular localization of chicken vimentin we performed double-label immunofluorescence microscopy on 3T3MTV ${ }^{2}-108$ cells following similar periods of induction and deinduction. Using double-label immunofluorescence microscopy, we detected no chicken vimentin-specific staining in uninduced cells (Fig. 3A), while the characteristic mouse vimentin filament array was apparent (Fig. 3B). After $4 \mathrm{~h}$ of dexamethasone induction, newly assembled chicken vimentin localized to discrete, irregular foci (Fig. 3C), which colocalized with the total vimentin filament network (Fig. 3D), in agreement with earlier work (Ngai et al., 1990). A careful examination of Fig. $3 \mathrm{C}$ and $\mathrm{G}$ reveals short interspersed segments of chicken vimentin staining, or a 'discontinuous' pattern, as opposed to the uniform filamentous array seen in Fig. 3E, which we refer to as a 'continuous' pattern. After a $4 \mathrm{~h}$ induction period, dexamethasone was removed and the cells were subsequently chased in the absence of inducer. When examined after a $9 \mathrm{~h}$ chase period the chicken vimentin staining had coalesced into a continuous filamentous array (Fig. 3E) that was coincident with the endogenous vimentin network (Fig. 3F). Interestingly, after longer chase periods in the absence of dexamethasone ( $28 \mathrm{~h}$ ), the strikingly irregular, discontinuous chicken vimentin distribution was apparent (Fig. 3G). This progression from discontinuous to continuous to discontinuous required protein synthesis, as cells induced for $4 \mathrm{~h}$ and then chased without dexamethasone in the presence of either cycloheximide $\left(10^{-6} \mathrm{M}\right)$ or emetine $\left(10^{-6} \mathrm{M}\right)$ were arrested in a discontinuous staining pattern independent of chase time (9-32 h; data not shown).

Though these cells are a permanent line (resulting from the stable transfection of the inducible chicken vimentin construct), the expression of chicken vimentin was not always homogeneous (Ngai et al., 1990); cells inducible for chicken vimentin expression represented anywhere from $30-95 \%$ of the population (compare Fig. 3C-F). Surprisingly, when subcloned by limiting dilution and reanalyzed for inducible chicken vimentin expression each subclone displayed a similar variable inducibility. The reasons for this phenotypic variation remain unknown but this phenomenon has been noted for other expression systems. Furthermore, as only chicken vimentin positive cells were scored and both the kinetics of induction/deinduction and the intracellular localization of chicken vimentin were qualitatively identical in these cells, this phenotypic variation did not affect the quantitation of our results.

We quantitated the progression of chicken vimentin immunolocalization for populations of cells. 3T3MTV -108 cells were induced with dexamethasone for $4 \mathrm{~h}$, chased for various times, fixed and stained for chicken and mouse vimentin immunofluorescence. We scored each chicken vimentin-positive cell as either discontinuous (comparable to Fig. 3C and G) or continuous (comparable to Fig. 3E). Multiple experiments consistently revealed that chicken vimentin staining in a significant proportion of cells did progress from discontinuous (if examined shortly after induction; 0-6 h) to continuous (if examined after 8-10 h chase) to discontinuous (if examined after $\geq 20 \mathrm{~h}$ chase; Fig. 4). Data from multiple induction/deinduction experiments 

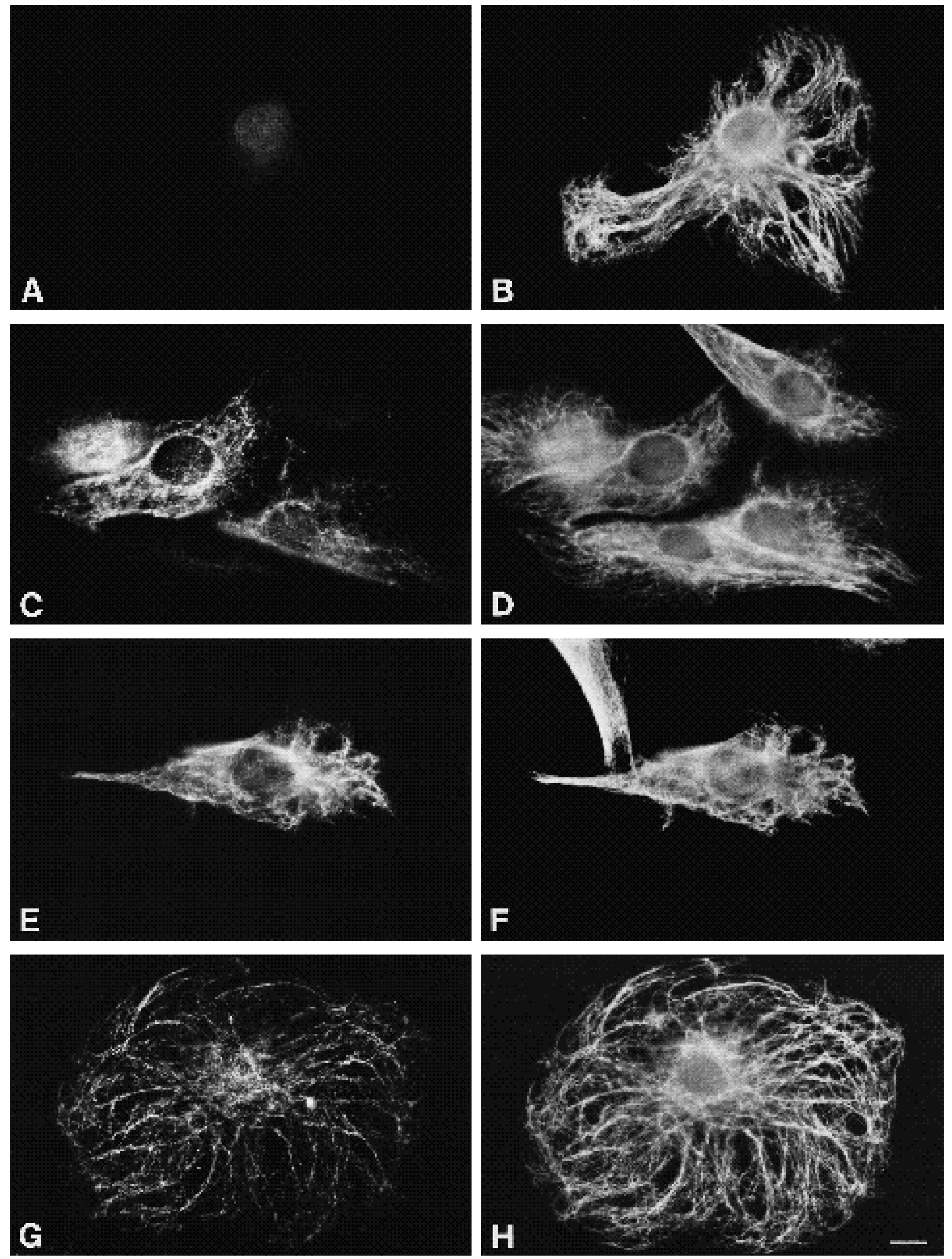

Fig. 3 
Fig. 3. Induction/deinduction experiment showing fate of induced chicken vimentin protein, after various times, in the absence of dexamethasone. 3T3MTV ${ }^{2}-108$ cells were fixed and processed for double-label immunofluorescence microscopy following incubation in the absence $(\mathrm{A}, \mathrm{B})$ or presence $(\mathrm{C}-\mathrm{H})$ of dexamethasone. All cells in $\mathrm{C}-\mathrm{H}$ were induced for $4 \mathrm{~h}$ then chased in the absence of dexamethasone for $0 \mathrm{~h}(\mathrm{C}, \mathrm{D}), 9 \mathrm{~h}(\mathrm{E}, \mathrm{F})$ and $28 \mathrm{~h}$ $(\mathrm{G}, \mathrm{H})$, respectively. Chicken vimentin was localized using monoclonal antibody 10D1/D11 (A,C,E,G). Total vimentin (mouse plus chicken) was detected using a goat polyclonal antivimentin antibody (B,D,F,H). Note the discontinuous chicken vimentin distribution in cells fixed directly after induction (C), which coalesces into a continuous staining pattern following a $9 \mathrm{~h}$ chase in the absence of dexamethasone (E), only to become discontinuous again after a $28 \mathrm{~h}$ chase period $(\mathrm{G})$. Fibroblast morphology varies greatly from cell to cell and these fields have been chosen strictly on the basis of vimentin staining. Each pair of micrographs was photographed at the same plane of focus; bar, 10 $\mu \mathrm{m}$.

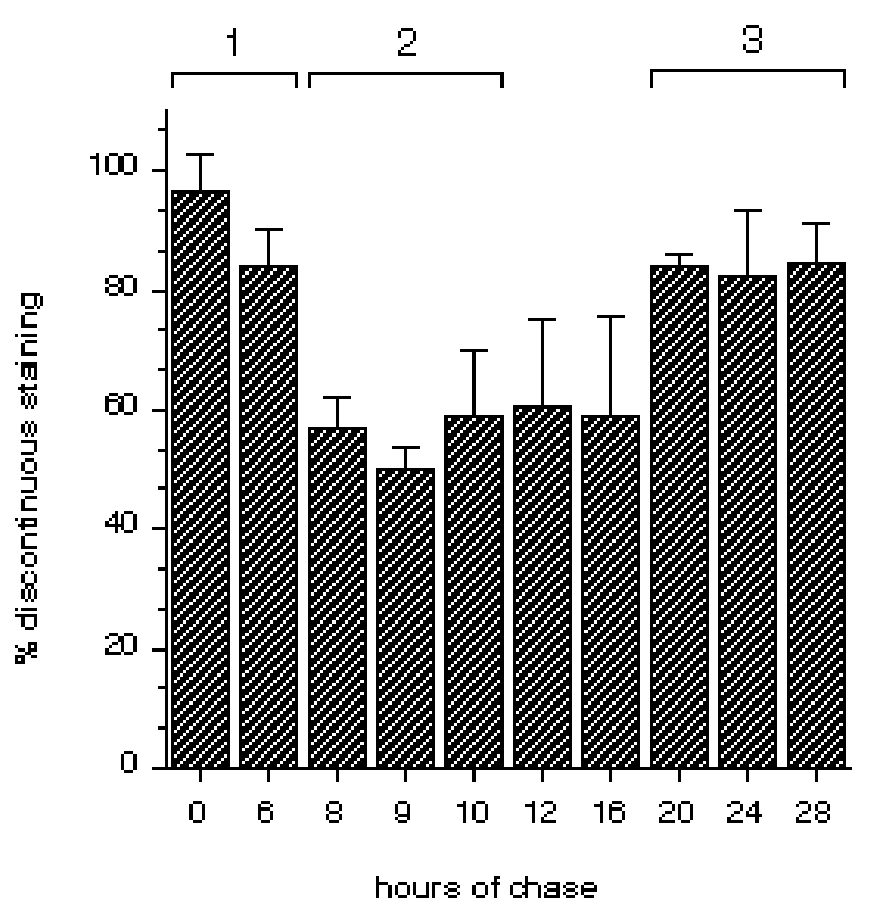

Fig. 4. Quantitation of multiple induction/deinduction experiments similar to that shown in Fig. 3. 3T3MTV²-108 cells were scored as either discontinuous or continuous and the data were plotted in histogram form as percentage discontinuous chicken vimentin staining. All cells were induced with dexamethasone for $4 \mathrm{~h}$ and chased in the absence of dexamethasone for the times indicated $(0-28 \mathrm{~h})$. Note how a significant proportion of the cells progress from a discontinuous chicken vimentin distribution pattern (if fixed shortly after induction) to a continuous staining pattern (if chased in the absence of dexamethasone, $8-10 \mathrm{~h}$ ) back to a discontinuous staining pattern (if chased in the absence of dexamethasone, 20-28 h). Data from the bracketed chase times (groups 1,2,3) were pooled and compared with one another using the Bonferroni multiple comparison procedure (see Results). Histogram bars represent an average of 140-1000 cells from 8 separate experiments; error bar indicates 1 s.d. were pooled, divided into three groups (Fig. 4, group 1, 0$6 \mathrm{~h}$ chase; group 2, 8-10 h chase; group $3, \geq 20 \mathrm{~h}$ chase) and compared with one another using the Bonferroni multiple comparison procedure (McClave and Dietrich, 1988). The discontinuous (groups 1 and 3) and continuous (group $2)$ differed significantly $(P<0.001)$. Groups 1 and 3 were not statistically different, consistent with our designating them both discontinuous staining.

Since these cells divide every $28 \mathrm{~h}$, the reappearance of discontinuous chicken vimentin immunofluorescence at later chase times could have been due to vimentin subunit rearrangements, which potentially take place during mitosis. To test the effects due to cell division, we established conditions which arrested cell division, yet allowed for dexamethasone inducibility. Drugs that interfere with DNA replication, such as cytosine arabinofuranoside, inhibited dexamethasone inducibility of the cells. Treatment with colchicine $\left(10^{-6} \mathrm{M}\right)$ caused the characteristic IF collapse into a cage around the nucleus, which prevented visualization of the discontinuous chicken vimentin staining. Preincubation of $3 \mathrm{~T}^{3} \mathrm{MTV}^{2}-108$ cells in low-serum $(0.5 \%)$ DMEM for $48 \mathrm{~h}$, however, both arrested cell division (as assayed by $\left[{ }^{3} \mathrm{H}\right]$ thymidine uptake over an additional $48 \mathrm{~h}$ period; Tucker et al., 1979) and allowed dexamethasoneinduction of chicken vimentin expression. Induction/deinduction experiments performed using these mitotically arrested cells revealed that chicken vimentin immunofluorescence staining progressed from a discontinuous to a continuous to a discontinuous pattern with identical kinetics to those of proliferating cells (data not shown). Thus, the discontinuous chicken vimentin immunofluorescence pattern, which reappeared following $\geq 20 \mathrm{~h}$ chase, cannot be attributed to mitotic-induced IF rearrangements; rather, this discontinuous pattern is a likely consequence of the general mechanism of interphase IF assembly.

\section{Discussion}

\section{Intermediate filaments grow continuously during} interphase

In this study we have monitored in vivo changes in vimentin mRNA kinetics, protein kinetics and immunolocalization following deinduction of a traceable vimentin subunit. We have demonstrated that IFs polymerize via subunit insertion at numerous discrete foci throughout the cytoplasm. Following removal of dexamethasone after a $4 \mathrm{~h}$ induction in 3T3MTV ${ }^{2}-108$ cells, chicken vimentin immunolocalization superimposed on the pre-existing filament network and progressed from a discontinuous ( $0-6 \mathrm{~h}$ chase) to a continuous (8-10 $\mathrm{h}$ chase) to a discontinuous ( $\geq 20 \mathrm{~h}$ chase) staining pattern. Using quantitative RNase protection we have determined that chicken vimentin mRNA has a half-life of $\sim 6 \mathrm{~h}$. Since vimentin expression is regulated primarily by mRNA abundance (Ngai et al., 1984), induced 3T3MTV2_ 108 cells would be expected to continue making significant chicken vimentin protein for an additional 8-10 h following dexamethasone removal. Thus the conversion from discontinuous ( $0-6 \mathrm{~h}$ chase) to continuous ( $8-10 \mathrm{~h}$ chase) staining was likely due to continued chicken vimentin protein synthesis and assembly through translation of residual 
chicken vimentin mRNA. After a 12-16 h chase, however, little chicken vimentin mRNA remained and by $\geq 20 \mathrm{~h}$ of chase $3 \mathrm{~T}_{3} \mathrm{MTV}^{2}-108$ cells had progressed back to discontinuous staining. Moreover, metabolic pulse-chase studies using $\left.{ }^{35} \mathrm{~S}\right]$ methionine revealed that both chicken and mouse vimentin protein were stable cytoskeletal components with half-lives approximating that of cell generation time, in agreement with earlier work (McTavish et al., 1983). Taken together, these data are most consistent with a continuously growing model of vimentin assembly. In such a model, the continuous (8-10 h chase) to discontinuous ( $\geq 20 \mathrm{~h}$ chase) chicken vimentin staining progression would be due to continued incorporation of newly synthesized mouse vimentin into the filamentous array in the absence of chicken vimentin mRNA and subunit synthesis. Over time, mouse vimentin subunits would incorporate at numerous sites throughout the continuously labeled chicken vimentin network, thereby separating it into a discontinuous pattern.

Using a similar inducible system, Sarria et al. (1990) have shown that induced mouse vimentin cDNA expression in HeLa cells results in incorporation of newly synthesized mouse vimentin into the pre-existing human filament network. In their system, however, they do not observe the punctate localization of newly synthesized vimentin along endogenous filaments that we report here (Fig. 3) and previously (Ngai et al., 1990). After removal of dexamethasone from the HeLa cells, the intensity of induced vimentin immunoreactivity slowly diminished but never revealed a punctate localization. The reason for these discrepancies is unknown, but may reflect a difference in the kinetics and/or number of IF subunit entry points for these two cell types. Significantly, previous immunoelectron microscopic analysis with 3T3MTV ${ }^{2}-108$ fibroblasts has confirmed that the punctate pattern of newly assembled chicken vimentin reflects the scattered incorporation of chicken vimentin within single mouse vimentin filaments (Ngai et al., 1990). While it is formally possible that the punctate immunofluorescence pattern is due to intersection of two or more chicken vimentin-containing filaments in one optical section, we think this is unlikely, particularly in view of our immunoelectron microscopic results (Ngai et al., 1990).

One interpretation of a relatively long IF half-life is that once IF subunits are incorporated into the polymer they do not disassemble, or do so infrequently such as during mitosis. Indeed, perhaps IFs are not turned over per se but rather halved by division (i.e. physically separated into the two daughter cells during cytokinesis). During subsequent interphase the daughter cells would add to their IF network until the next division. The time course of our pulse-chase studies, however, is not inconsistent with the subunit exchange in and out of the IF polymer seen in several laboratories. For example, in vitro fluorescence resonance energy-transfer studies have demonstrated that the neurofilament polymer is in dynamic equilibrium with its subunit pool (Angelides et al., 1989). Similarly, studies monitoring fluorescence recovery after photobleaching in cells injected with xrhodamine-vimentin demonstrate exchange in and out of the IF polymer (Vikstrom et al., 1992). These data suggest that, once assembled, IF subunits can exit from the polymer; within the resolution limits of our pulse-chase experiments, however, if incorporated subunits do exit from the polymer they do so transiently.

\section{De novo IF formation versus incorporation into pre- existing IF network}

Two possible mechanisms for the maintenance/growth of IF network exist: the formation of new IF polymers and/or the elongation of pre-existing filaments. A body of evidence favors de novo assembly of IFs initiating from the nucleus. For example, soluble IF subunits polymerize from isolated nuclei (Eckert et al., 1982; Wang, 1985) and lamin B may represent one nuclear IF binding site (Georgatos and Blobel, 1987a,b). Moreover, microinjected biotinylated vimentin slowly progresses from a perinuclear region to the characteristic filamentous array (Vikstrom et al., 1989). Finally, following keratin filament disruption, recovery was observed to occur in close proximity to the nucleus (Albers and Fuchs, 1987). Our results, however, suggest that de novo nucleus-associated IF assembly is not the only means of filament growth. If vimentin assembly were a polarized process directed outward from the nucleus, then upon removal of inducer, it would be anticipated that chicken vimentin would be preferentially displaced from the perinuclear region as newly assembled mouse vimentin was incorporated. Since we and others (Sarria et al., 1990) do not observe such a polarized process, our results are most consistent with an IF assembly mechanism whereby newly synthesized subunits are incorporated into the pre-existing IF network at foci throughout the entire cytoplasm. Such a model is also consistent with the nonpolar fluorescence recovery of xrhodamine-vimentin fibers following photobleaching (Vikstrom et al., 1992) and the work of Miller et al. (1991), who found rapid subunit incorporation at numerous sites along the length of the endogenous tonofilament (keratin-containing intermediate filament) bundles.

This notion of restricted foci of IF assembly on pre-existing filaments suggests the existence of discrete assemblycompetent zones along filaments. These specialized-assembly zones may contain essential IF binding proteins. In light of recent evidence that vimentin filament assembly may proceed cotranslationally, these assembly zones may, in fact, contain cytoskeletal-associated vimentin-translating ribosomes (Isaacs et al., 1989). If assembly does occur at interior sites along the filament contour, a transient disruption of filament or subfilament integrity would be necessary to allow subunit entry (see Ngai et al., 1990). New subunits may be added onto the sides of filaments as the first step before actual integration. Such lateral associations are consistent with the variable mass per length found for many IFs and may correlate with variable numbers of 4.5 $\mathrm{nm}$ protofibrils per filament cross-section (Engel et al., 1985).

\section{Implications of IF subunit replacement and stable IF networks}

A variety of cells undergo IF subunit-switching during differentiation (e.g. vimentin to neurofilament: Tapscott et al., 1981; vimentin to desmin: Bennett et al., 1979; Gard and Lazarides, 1980; and vimentin to GFAP: Tapscott et al., 1981; Schnitzer et al., 1981; Yen and Fields, 1981). The mechanism by which IF subunit-switching occurs is not 
completely understood and is particularly interesting in view of the fact that wherever GFAP, vimentin or neurofilament proteins are coexpressed, random heteropolymers form (Gard et al., 1979; Steinert et al., 1981; Quinlan and Franke, 1982, 1983; Sharp et al., 1982; Granger and Lazarides, 1983; Ip et al., 1983; Monteiro and Cleveland, 1989). The present study suggests that during IF subunitswitching, vimentin protein is "lost" via dilution of existing filaments rather than by an active catabolic mechanism. Moreover, since newly synthesized IF subunits insert at discrete sites throughout the pre-existing filament network, the topography of this IF protein type switch likely mirrors our induction/deinduction experiments, wherein a homogeneous polymer (equivalent to continuously stained) converts to a heterogeneous polymer (equivalent to discontinuously stained) by insertional dilution. Interestingly, immunoelectron microscopy of cells which naturally coexpress two IF protein types has revealed that individual IFs have discrete incorporation sites (Sharp et al., 1982; Ip et al., 1983). Since a similar discontinuous immunoelectron staining pattern is also seen in our transfected cells upon induction (Ngai et al., 1990), it seems likely that this punctate topography of assembly reflects the mechanistically similar process of differentiation-induced IF protein-type switching in vivo. Over time, in the absence of old IF protein type synthesis, the differentiation-induced insertional dilution would result in the new IF protein type replacing the pre-existing filament network.

The authors thank the other members of the Lazarides laboratory for their critical input and many useful discussions. Special thanks to Dr. Frank Sangiorgi for his RNase protection tutelage and Alexander Kratz (Yale University) for his statistical expertise. We also warmly thank Drs. William Dunphy (Caltech), Sarah Fashena (Yale University), Douglas Fishkind (Worcester Foundation of Experimental Biology), John Ngai (Columbia University) and Barbara Wold (Caltech) for their helpful discussions and comments on the manuscript. This work was supported by grants from the National Institutes of Health (AG 06078A). T.R.C. was supported by a postdoctoral fellowship from the Damon RunyonWalter Winchell Cancer Research Fund (DRG-990).

\section{References}

Albers, K. and Fuchs, E. (1987). The expression of mutant epidermal keratin cDNAs transfected in simple epithelial and squamous cell carcinoma lines. J. Cell Biol. 105, 791-806.

Albers, K. and Fuchs, E. (1989). Expression of mutant keratin cDNAs in epithelial cells reveals possible mechanisms for initiation and assembly of intermediate filaments. J. Cell Biol. 108, 1477-1493.

Angelides, K. J., Smith, K. E. and Tokeda, M. (1989). Assembly and exchange of intermediate filament proteins of neurons: Neurofilaments are dynamic structures. J. Cell Biol. 108, 1495-1506.

Bradford, M. M. (1976). A rapid and sensitive method for the quantitation of microgram quantities of protein utilizing the principle of protein-dye binding. Anal. Biochem. 72, 248-254.

Bennett, G. S., Fellini, S. A., Toyama, Y. and Holtzer, H. (1979). Redistribution of intermediate filament subunits during skeletal myogenesis and maturation in vitro. J. Cell Biol. 82, 577-584.

Blikstad, I. and Lazarides, E. (1983). Vimentin filaments are assembled from a soluble precursor in avian erythroid cells. J. Cell Biol. 96, 18031808.

Bonner, W. M. and Laskey, R. A. (1974). A film detection method for tritium-labelled proteins and nucleic acids in polyacrylamide gels. Eur. J. Biochem. 46, 83-88.

Chirgwin, J. M., Przybyla, A. E., MacDonald, R. J. and Rutter, W. J. (1979). Isolation of biologically active ribonucleic acid from sources enriched in ribonuclease. Biochemistry 18, 5294-5299.

Chou, Y.-H., Bischoff, J. R., Beach, D. and Goldman, R. D. (1990). Intermediate filament reorganization during mitosis is mediated by p34 ${ }^{\mathrm{cdc} 2}$ phosphorylation of vimentin. Cell 62, 1063-1071.

Christian, J. L., Edelstein, N. G. and Moon, R. T. (1990). Overexpression of wild-type and dominant negative mutant vimentin subunits in developing Xenopus embryos. The New Biologist 2, 700-711.

Coleman, T. R., Harris, A. S., Mische, S. M., Mooseker, M. S. and Morrow, J. S. (1987). Beta spectrin bestows protein 4.1 sensitivity on spectrin-actin interactions. J. Cell Biol. 104, 519-526.

Eckert, B. S., Daley, R. A. and Parysek, L. M. (1982). Assembly of keratin onto $\mathrm{PtK}_{1}$ cytoskeletons: Evidence for an intermediate filament organizing center. J. Cell Biol. 92, 575-578.

Engel, A., Eichner, R. and Aebi, U. (1985). Polymorphism of reconstituted human epidermal keratin filaments: Determination of their mass-perlength and width by scanning transmission electron microscopy (STEM). J. Ultrastruct. Res. 90, 323-335.

Evans, R. M. (1988a). Cyclic AMP-dependent protein kinase-induced vimentin filament disassembly involves modification of the N-terminal domain of intermediate filament subunits. FEBS Lett. 234, 73-78.

Evans, R. M. (1988b). The intermediate-filament proteins vimentin and desmin are phosphorylated in specific domains. Eur. J. Cell Biol. 46, 152160.

Gard, D. L., Bell, P. B. and Lazarides, E. (1979). Coexistence of desmin and the fibroblastic intermediate filament subunit in muscle and nonmuscle cells: Identification and comparative peptide analysis. Proc. Nat. Acad. Sci. USA 76, 3894-3898.

Gard, D. L. and Lazarides, E. (1980). The synthesis and distribution of desmin and vimentin during myogenesis in vitro. Cell 19, 263-275.

Geisler, N. and Weber, K. (1988). Phosphorylation of desmin in vitro inhibits formation of intermediate filaments; identification of three kinase A sites in the aminoterminal head domain. EMBO J. 7, 15-20.

Georgatos, S. D. and Blobel, G. (1987a). Lamin B constitutes an intermediate filament attachment site at the nuclear envelope. J. Cell Biol. 105, 117-125.

Georgatos, S. D. and Blobel, G. (1987b). Two distinct attachment sites for vimentin along the plasma membrane and the nuclear envelope in avian erythrocytes: A basis for a vectorial assembly of intermediate filaments. $J$. Cell Biol. 105, 105-115.

Gerace, L. and Blobel, G. (1980). The nuclear envelope lamina is reversibly depolymerized during mitosis. Cell 19, 277-287.

Gill, S. R., Wong, P. C., Monteiro, M. J. and Cleveland, D. W. (1990). Assembly properties of dominant and recessive mutations in the small mouse neurofilament (NF-L) subunit. J. Cell Biol. 111, 2005-2019.

Granger, B. L. and Lazarides, E. (1983). Expression of the major neurofilament subunit in chicken erythrocytes. Science 221, 553-556.

Granger, B. L., Repasky, E. A. and Lazarides, E. (1982). Synemin and vimentin are components of intermediate filaments in avian erythrocytes. J. Cell Biol. 92, 299-312.

Heald, R. and McKeon, F. (1990). Mutations of phosphorylation sites in lamin A that prevent nuclear lamina disassembly in mitosis. Cell 61, 579589.

Hisanaga, S.-I., Gonda, Y., Inagaki, M., Ikai, A. and Hirokawa, N. (1990). Effects of phosphorylation of the neurofilament L protein on filamentous structures. Cell Reg. 1, 237-248.

Inagaki, M., Gonda, Y., Matsuyama, M., Nishizawa, K., Nishi, Y. and Sato, C. (1988). Intermediate filament reconstitution in vitro. J. Biol. Chem. 263, 5970-5978.

Inagaki, M., Nishi, Y., Nishizawa, K., Matsuyama, M. and Sato, C. (1987). Site-specific phosphorylation induces disassembly of vimentin filaments in vitro. Nature 328, 649-652.

Ip, W., Danto, S. I. and Fischman, D. (1983). Detection of desmincontaining intermediate filaments in cultured muscle and nonmuscle cells by immunoelectron microscopy. J. Cell. Biol. 96, 401-408.

Isaacs, W. B., Cook, R. K., Van Atta, J. C., Redmond, C. M. and Fulton, A. B. (1989). Assembly of vimentin in cultured cells varies with cell type. J. Biol. Chem. 264, 17953-17960.

Klymkowsky, M. W., Bachant, J. B. and Domingo, A. (1989). Functions of intermediate filaments. Cell Motil. Cytoskel. 14, 309-331.

Lamb, N. J. C., Fernandez, A., Feramisco, J. R. and Welch, W. J. (1989). 
Modulation of vimentin containing intermediate filament distribution and phosphorylation in living fibroblasts by the cAMP-dependent protein kinase. J. Cell Biol. 108, 2409-2422.

Lu, X. and Lane, E. B. (1990). Retrovirus-mediated transgenic keratin expression in cultured fibroblasts: Specific domain functions in keratin stabilization and filament formation. Cell 62, 681-696.

McClave, J. T. and Dietrich, F. H. (1988). Statistics, fourth edn, p. 486 Dellen Publishing Company, San Francisco, CA.

McTavish, C. F., Nelson, W. J. and Traub, P. (1983). The turnover of vimentin in Ehrlich ascites tumour cells. FEBS Lett. 154, 251-256.

Miller, R. K., Vikstrom, K. and Goldman, R. D. (1991). Keratin incorporation into intermediate filament networks is a rapid process. $J$. Cell Biol. 113, 843-855.

Monteiro, M. J. and Cleveland, D. W. (1989). Expression of NF-L and NF-M in fibroblasts reveals coassembly of neurofilament and vimentin subunits. J. Cell Biol. 108, 579-593.

Moon, R. T. and Lazarides, E. (1983). Synthesis and post-translational assembly of intermediate filaments in avian erythroid cells: Vimentin assembly limits the rate of synemin assembly. Proc. Nat. Acad. Sci. USA 80, 5495-5499.

Nelson, W. J. and Traub, P. (1982). Intermediate (10 nm) filament proteins and the $\mathrm{Ca}^{2+}$-activated proteinase specific for vimentin and desmin in the cells from fish to man: An example of evolutionary conservation. J. Cell Sci. 57, 25-49.

Ngai, J., Bond, V. C., Wold, B. J. and Lazarides, E. (1987). Expression of transfected vimentin genes in differentiating murine erythroleukemia cells reveals divergent cis-acting regulation of avian and mammalian vimentin sequences. Mol. Cell. Biol. 7, 3955-3970.

Ngai, J., Capetanaki, Y. G. and Lazarides, E. (1984). Differentiation of murine erythroleukemia cells results in the rapid repression of vimentin gene expression. J. Cell Biol. 99, 306-314.

Ngai, J., Coleman, T. R. and Lazarides, E. (1990). Localization of newly synthesized vimentin subunits reveals a novel mechanism of intermediate filament assembly. Cell 60, 415-427.

Peter, M., Nakagawa, J., Dorée, M., Labbé, J. C. and Nigg, E. A. (1990). In vitro disassembly of the nuclear lamina and $\mathrm{M}$ phase-specific phosphorylation of lamins by cdc2 kinase. Cell 61, 591-602.

Quinlan, R. A. and Franke, W. W. (1982). Heteropolymer filaments of vimentin and desmin in vascular smooth muscle tissue and cultured baby hamster kidney cells demonstrated by chemical crosslinking. Proc. Nat. Acad. Sci. USA 79, 3452-3456.

Quinlan, R. A. and Franke, W. W. (1983). Molecular interactions in intermediate-sized filaments revealed by chemical cross-linking. Eur. J. Biochem. 132, 477-484.

Raats, J. M. H., Pieper, F. R., Vree Egberts, W. T. M., Verrijp, K. N., Ramaekers, F. C. S. and Bloemendal, H. (1990). Assembly of aminoterminally deleted desmin in vimentin-free cells. J. Cell Biol. 111, 19711985.

Sambrook, J., Fritsch, E. F. and Maniatis, T. (1989). Molecular Cloning, A Laboratory Manual, second edn. Cold Spring Harbor Laboratory Press, NY.
Sarria, A. J., Nordeen, S. K. and Evans, R. M. (1990). Regulated expression of vimentin cDNA in cells in the presence and absence of a preexisting vimentin filament network. J. Cell Biol. 111, 553-565.

Schnitzer, J., Franke, W. W. and Schachner, M. (1981). Immunocytochemical demonstration of vimentin in astrocytes and ependymal cells of developing and adult mouse nervous system. J. Cell Biol. 90, 435-447.

Sharp, G., Osborn, M. and Weber, K. (1982). Occurrence of two different intermediate filament proteins in the same filament in situ within a human glioma cell line. Exp. Cell Res. 141, 385-395.

Skalli, O. and Goldman, R. D. (1991). Recent insights into the assembly, dynamics and function of intermediate filament networks. Cell Motil. Cytoskel. 19, 67-79.

Spudich, J. A. and Watt, S. (1971). The regulation of rabbit skeletal muscle contraction. J. Biol. Chem. 246, 4806-4871.

Steinert, P. M., Ider, W. W., Cabral, F., Gottesman, M. M. and Goldman, R. D. (1981). In vitro assembly of homopolymer and copolymer filaments from intermediate filament subunits of muscle and fibroblastic cells. Proc. Nat. Acad. Sci. USA 78, 3692-3696.

Steinert, P. M. and Roop, D. R. (1988). Molecular and cellular biology of intermediate filaments. Аnпи. Rev. Biochem. 57, 593-625.

Steinert, P. M. and Liem, R. K. H. (1990). Intermediate filament dynamics. Cell 60, 521-523.

Stewart, M. (1990). Intermediate filaments: structure, assembly and molecular interactions. Curr. Opin. Cell Biol. 2, 91-100.

Tapscott, S. J., Bennett, G. S. and Holtzer, H. (1981). Neuronal precursor cells in the chick neural tube express neurofilament proteins. Nature 292, 836-838.

Tucker, R. W., Pardee, A. B. and Fujiwara, K. (1979). Centriole ciliation is related to quiescence and DNA synthesis in 3T3 cells. Cell 17, 527-535.

Vikstrom, K. L., Borisy, G. G. and Goldman, R. D. (1989). Dynamic aspects of intermediate filament networks in BHK-21 cells. Proc. Nat. Acad. Sci. USA 86, 549-553.

Vikstrom, K. L., Lim, S. S., Goldman, R. D. and Borisy, G. G. (1992). Steady state dynamics of intermediate filament networks. J. Cell Biol. 118, 121-129.

Wang, E. (1985). Intermediate filament associated proteins. Ann N.Y. Acad. Sci. 455, 32-56.

Ward, G. E. and Kirschner, M. W. (1990). Identification of cell cycleregulated phosphorylation sites on nuclear lamin C. Cell 61, 561-577.

Wong, P. C. and Cleveland, D. W. (1990). Characterization of dominant and recessive assembly-defective mutations in mouse neurofilament NFM. J. Cell Biol. 111, 1987-2003.

Yen, S.-H. and Fields, K. L. (1981). Antibodies to neurofilament, glial filament and fibroblast intermediate filament proteins bind to different cell types of the nervous system. J. Cell Biol. 88, 115-126.

Zinn, K., DiMaio, D. and Maniatis, T. (1983). Identification of two distinct regulatory regions adjacent to the human $\beta$-interferon gene. Cell 34, 865-879.

(Received 21 May 1992 - Accepted 24 July 1992) 